Vol.01/ No. 02

Pages: 91- 102

http://irojournals.com/jscp/

DOI: https://doi.org/10.36548/jscp.2019.2.004

\title{
QOS ENHANCED ROUTING PROTOCOLS FOR VEHICULAR NETWORK USING SOFT COMPUTING TECHNIQUE
}

\author{
Dr. Wang Haoxiang, \\ Director and lead executive faculty member, \\ GoPerception Laboratory, NY, USA \\ Email id: hw496@goperception.com. \\ Dr. S. Smys, \\ Professor, Department of CSE, \\ RVS Technical Campus, \\ Coimbatore, India. \\ Email id: smys375@gmail.com
}

\begin{abstract}
The vehicular network that scopes to reach the internet access to make use of the available service on road along with the improvement in the safety and convenience while travelling has become a very popular area of research as they lay as the foundation for the intelligent transportation system. But due to the high mobility of the vehicles the network topology remains dynamic and the connectivity between the vehicles keep on changing reducing the life expectancy of the links and failures in the delivery of the services. So this paves way for the entailment of the more effective routing protocol that would heighten the vehicular network quality of service. The proposed method in the paper puts forth the modified Ant colony optimization that integrates the clustering technique to frame optimal shortest route for the internet access in the vehicular network to manage its mobility and the topology changes. The process of optimal route selection taking into consideration the mobility and the dynamic topology is evaluated using the network simulator tool to prove the capabilities of the proposed method on the grounds of bandwidth utilization throughput delay and link life expectancy.
\end{abstract}

Keywords: Vehicular Network, Soft Computing, Evolutionary Algorithm, Ant Colony Optimization, Clustering, Quality of Service.

ISSN: 2582-2640 (online) 
Journal of Soft Computing Paradigm (JSCP) (2019)

Vol.01/ No. 02

Pages: 91- 102

http://irojournals.com/jscp/

DOI: https://doi.org/10.36548/jscp.2019.2.004

\section{INTRODUCTION}

The vehicular-Adhoc networks [1] are infrastructure less wireless communication network framed for a specific reason. It utilizes the volunteering vehicles as the nodes to extend communication between the vehicles. The node in the network takes up either the role of source/destination or acts the repeaters/relay nodes retransmitting the information transmitted from the source or the destination. The volatile network framed among the vehicles in the road deliver the ways to enhance the road safety by establishing co-operative applications for driving. [2]

The VANET's are a superior type of mobile adhoc networks that is very useful in improving convenience in travelling by providing the complete details of the traffic, blockages, pot holes, and natural disasters on road. They also enable the user to have the full details of the route availability that reduce the time in travelling along with the parking availability, nearby stay, fuel stations available etc. [3].

They communication in the vehicular-Adhoc network takes place in two ways as vehicle to vehicle communication and vehicle to/from road side unit communication.

Though enriched with numerous of advantages the vehicular networks suffer from dynamic topologies due to its high mobility resulting in the link failures causing an improper communication or wrong communication or delayed communication leading fatalities on road.

The attributes of the VANET, results in a reducing the quality of the service, in conveyance between the vehicle, as the quality of service is a very important entailment in the vehicular networks to enhances the performance in terms of throughput, end to end delay and link expectancy. So the paper puts for the soft computing approaches to enhance the quality of the service. The proposed method utilizes the modified Ant colony optimization that integrates the clustering technique to frame optimal shortest route for the internet access in the vehicular network that is very effective in managing the mobility and the topology changes of the network along with the timely information delivery to the vehicles on road improving the travel convenience and safety.

ISSN: 2582-2640 (online)

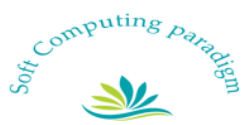


Journal of Soft Computing Paradigm (JSCP) (2019)

Vol.01/ No. 02

Pages: 91- 102

http://irojournals.com/jscp/

DOI: https://doi.org/10.36548/jscp.2019.2.004

The remaining paper is composed with the 2. Related works, 3. Proposed Routing Protocol, 4. Result Analysis and 5. Conclusion followed by references.

\section{RELATED WORKS}

The vehicular- adhoc networks being an special type of mobile adhoc networks the paper routing methods remains almost the same so the related works also includes the researches that have proceeded with the aim of developing a QOS Optimized routing protocols for the wireless adhoc networks. "Simplified QoS factor for the adhoc network using fuzzy technique" was proposed by Zaghar et al [1], the author Toutouh et al [2] detailed the . "Parallel swarm intelligence in VANETs optimization" for having a routing protocol that enhances the performance of the VANET. Kaur et al [3] in his paper puts forth an "AODV extension using genetic algorithm for VANET"

Sharawi et al [4] presents the survey on the ""Routing wireless sensor networks based on soft computing paradigms" while Jaspal ety al [5] in his paper formulates the "Fuzzy Improved Genetic Approach for Route Optimization in MANET" and Giri, et al [6] in his paper elaborates the "Survey on Soft Computing Techniques for MultiConstrained QoS Routing in MANET"

Vijayakumar et al [7] proffers a "Research on QoS aware dynamic reconfiguration and performance measures in VANET" Nancharaiah, et al [8] puts forward the "Hybrid optimization using ant colony optimization and cuckoo search in MANET routing." Baskaran et al [9] presents the algorithm based on the "Fuzzy optimized and bee inspired for developing an improved QOS in the MANET"

Benamar et al [10] describes the "routing protocols available for the delay tolerant vehicular networks as a comprehensive survey" Kumar et al [11] provides the "comparative study of Various Routing Protocols in VANET." Kaur, et al [12] details the study on the "Reliable Routing Protocols for Vehicular Ad hoc Networks" Bhalaji et al [13] details the "Performance Evaluation of Flying Wireless Network with Vanet Routing Protocol."

ISSN: 2582-2640 (online)

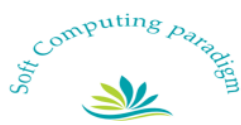


Journal of Soft Computing Paradigm (JSCP) (2019)

Vol.01/ No. 02

Pages: 91- 102

http://irojournals.com/jscp/

DOI: https://doi.org/10.36548/jscp.2019.2.004

Raj, Jennifer Set al [14] in her paper provides the "Energy Efficient Multi-Tier Sustainable Secure Routing Protocol for Mobile Wireless Sensor Networks" and Hoang et al [15] puts forward the "Realization of a cluster-based protocol using fuzzy C-means algorithm for wireless sensor networks."

\section{PROPOSED ROUTING PROTOCOL}

The paper puts forth the modified Ant colony based optimization that integrates the fuzzy $\mathrm{C}$ means clustering, to identify the optimal routing between the vehicles s to enhance the QOS metrics such as the throughput, end to end delay and life expectancy link. The proposed method proceeds as two stages such as the

(i). Cluster Framing, Cluster Head Selection and Relay Member Selection

(ii) Optimal Route Selection

\section{(i). CLUSTER FRAMING AND CLUSTER HEAD SELECTION}

The proposed method utilizes the fuzzy $\mathrm{C}$ means clustering [15] to frame the cluster enumerating the distance (dist ${ }_{\text {nodes }}$ ) of the nodes, the mobility of the nodes and the cluster heads are selected based on the metric such as the bandwidth availability $\left(B W_{\text {availbility }}\right)$, delay $\left(D_{E-E}\right)$, mobility $\left(M_{\text {nodes }}\right)$ and the degree of the nodes $\left(D e g_{n}\right)$. The volunteering nodes with the minimum dist $_{\text {nodes }}$ and the $M_{\text {nodes }}$ are identified and framed as the clusters and the cluster with the maximum $B W_{\text {availbility }}$, minimum delay $\left(D_{E-E}\right)$, mobility $\left(M_{\text {nodes }}\right)$ and $D e g_{n}$ are selected as the cluster head. In order to reduce the information flooding in between and within the clusters during the information conveyance the certain nodes in the clusters are selected as the relay nodes. The flow chart below in the fig .1 explains the cluster framing $\left(C_{f}\right)$, cluster-head selection $\left(\mathrm{CH}_{s}\right)$ and relay member selection $\left(R M_{S}\right)$.

ISSN: 2582-2640 (online) 
Vol.01/ No. 02

Pages: 91- 102

http://irojournals.com/jscp/

DOI: https://doi.org/10.36548/jscp.2019.2.004

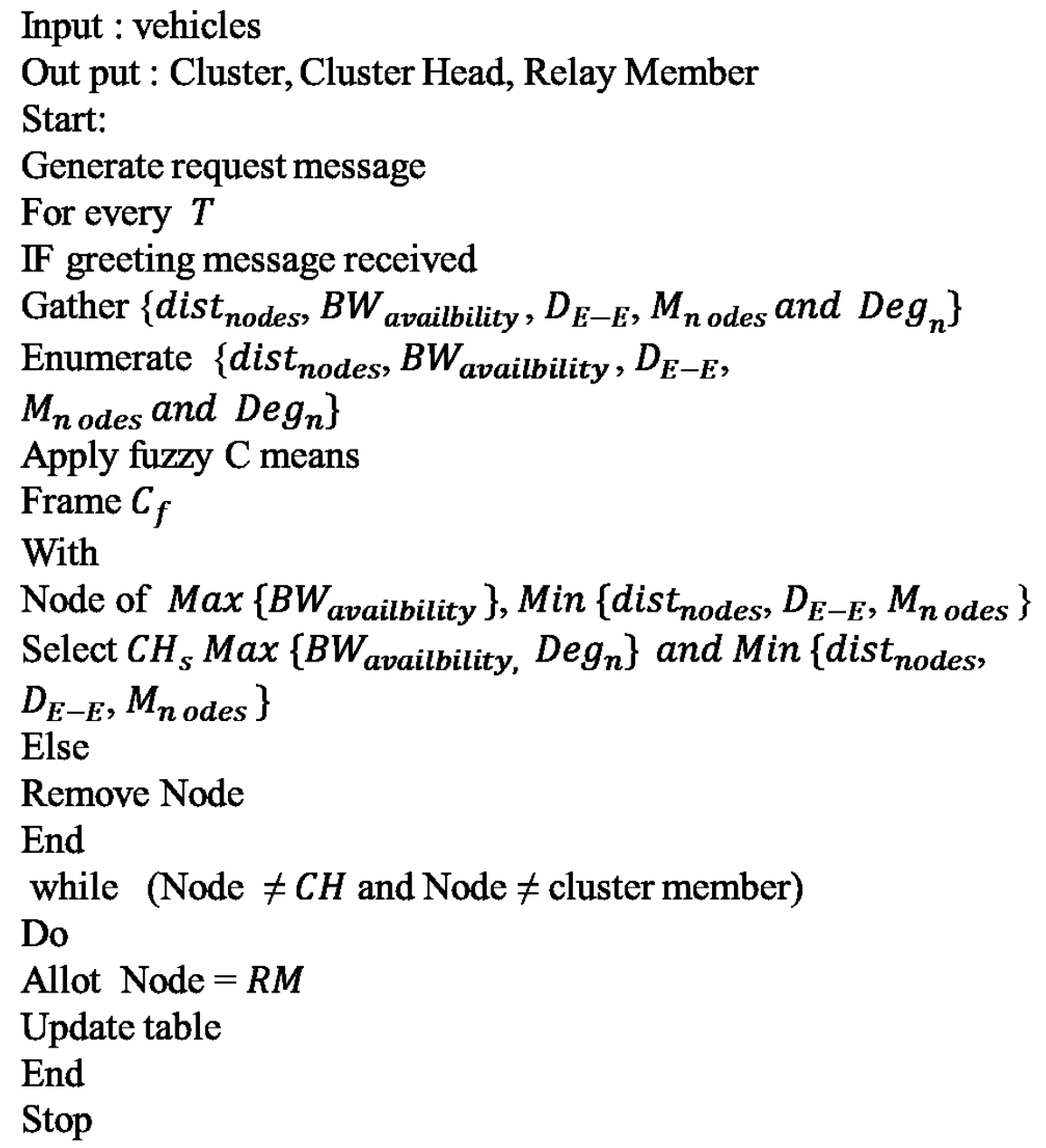

Fig.1 Flow Chart for Cluster Framing, Cluster Head Selection and Relay Member Selection

The greeting message is sent on regular intervals $(T)$ between the vehicles intimating their presence inside the cluster. The failure to send the greeting messages would be considered that the vehicle has left the cluster. Based on the greeting s received the list of the neighbors or the members of the cluster are updated to the neighbor list table periodically. Once the clusters are framed and the head is elected followed by the relay member selection. The optimal route for the conveyance is identified enumerating the vehicles that are very near to the destination, and identifying the vehicles with the maximum $B W_{\text {availbility }}$, minimum delay $\left(D_{E-E}\right)$, mobility $\left(M_{\text {nodes }}\right)$. 
Journal of Soft Computing Paradigm (JSCP) (2019)

Vol.01/ No. 02

Pages: 91- 102

http://irojournals.com/jscp/

DOI: https://doi.org/10.36548/jscp.2019.2.004

\section{(ii). OPTIMAL ROUTE DISCOVERY AND SELECTION USING ANT COLONY OPTIMIZATION}

The proposed method utilizes the for-aging behavior of the ants to identify the optimal routes for the conveying the information's from the source to the destination. The ant colony optimization [8] algorithm is engaged to enumerate the vehicles with the maximum $B W_{\text {availbility }}$, minimum delay $\left(D_{E-E}\right)$, mobility $\left(M_{\text {nodes }}\right)$ to discover the optimal route and select the route.

Whenever a vehicles wants to convey information to another vehicle in a cluster that is beyond its line of sight. The source node initially conveys the information to the cluster-head $(C-H)$, immediately the $C-H$ checks for the available route and initiates the transmission if the route is available and initiates a message $\left(I_{M}\right)$ to discover a route if no route is available satisfying the quality of service criteria. The $I_{M}$ is transmitted to the destination through the $R M$. Once the $I_{M}$ reaches the destinations, trace of all the paths are sent to the source $C-H$ and the path with the maximum $B W_{\text {availbility }}$, minimum $D_{E-E}, M_{\text {nodes }}$ and dist $t_{\text {nodes }}$ is identified applying the Ant colony optimization and the information is conveyed. The paths enumerate are updated into the table that is initialized for routing and the information are conveyed.

The algorithm below provides the process involved in the discovering of routes and selection of the routes utilizing the ant colony optimization.

- Consider a set of clusters $C_{l}=\left\{C_{1}, C_{2} \ldots \ldots . C_{10}\right\}$, where each cluster $C_{i}=\left\{n_{1, \ldots \ldots \ldots . . . .} n_{n}\right\}$, in which one of the node is elected as the $C-H$, and few nodes are selected as the $R M$, for a transmission to take place from the node $n_{1_{C_{i}}}$ to $n_{2 C_{j}}$ the $n_{1_{C_{i}}}$ convey the information to the $C-H_{C_{i}}$,

- The $C-H_{C_{i}}$ searches for the path availability with the optimal QOS criteria, IF a path is found it immediately starts conveying the information.

- ELSE, the $C-H_{C_{i}}$ initiates the $I_{M}$ to neighboring clusters through the $R M$, and the route through which the $I_{M}$ are relayed is saved back in the table for routing.

- When the $C-H_{C_{j}}$ is reached the trace of all the paths are send back to the $C-H_{C_{i}}$ along with the QOS values of the path.

ISSN: 2582-2640 (online)

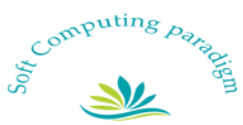


Journal of Soft Computing Paradigm (JSCP) (2019)

Vol.01/ No. 02

Pages: 91- 102

http://irojournals.com/jscp/

DOI: https://doi.org/10.36548/jscp.2019.2.004

- The path enriched with the maximum $B W_{\text {availbility }}, \operatorname{minimum} D_{E-E}, M_{\text {nodes }}$ and dist $t_{\text {nodes }}$ is enumerated applying the ant colony optimization and updated to the table

- The optimal path updated is utilized by the $C-H_{C_{i}}$ to convey the information form the $n_{1_{C_{i}}}$ to the $n_{2} C_{j}$ and STOPS.ELSE continues once again with the $I_{M}$ initiation to discover the optimal path.

The clustering methods based on the fuzzy $\mathrm{C}$ means and the discovery of the routes based on the ant colony optimization enables to the vehicular network to have a QOS optimized routing protocol that enhances the performance of the vehicular-Adhoc network in terms of the throughput, link life expectancy and delay.

\section{RESULT ANALYSIS}

The network simulator-2 evaluates the performance of the proposed protocol that presents a modified-ACO by incorporating the fuzzy means clustering to enumerate the QOS optimized path for the vehicular-Adhoc network , for the number of vehicles varying from 100 to 500 with a maximum speed not exceeding $40 \mathrm{~m} / \mathrm{s}$ within a transmission range of $250 \mathrm{~m}$. The evaluation is done on the $100 \mathrm{~m}$ road length which has two lanes for a simulation time of 1000s. The initial energy used in transmission for each node is recorded as 100 joules and the maximum packets to be transmitted per second are 1024 bits.

The fig .2 below shows the Throughput achieved by the proposed method for different number of vehicles for a simulation time s of 1000 seconds. The Throughput evaluation of the proposed method is compared with the other methods HACO-CS [8], ADOV-GA [3], PSO-V [2] and was found that the proposed method shows a heightened throughput than the prevailing methods.

ISSN: 2582-2640 (online) 
Journal of Soft Computing Paradigm (JSCP) (2019)

Vol.01/ No. 02

Pages: 91- 102

http://irojournals.com/jscp/

DOI: https://doi.org/10.36548/jscp.2019.2.004

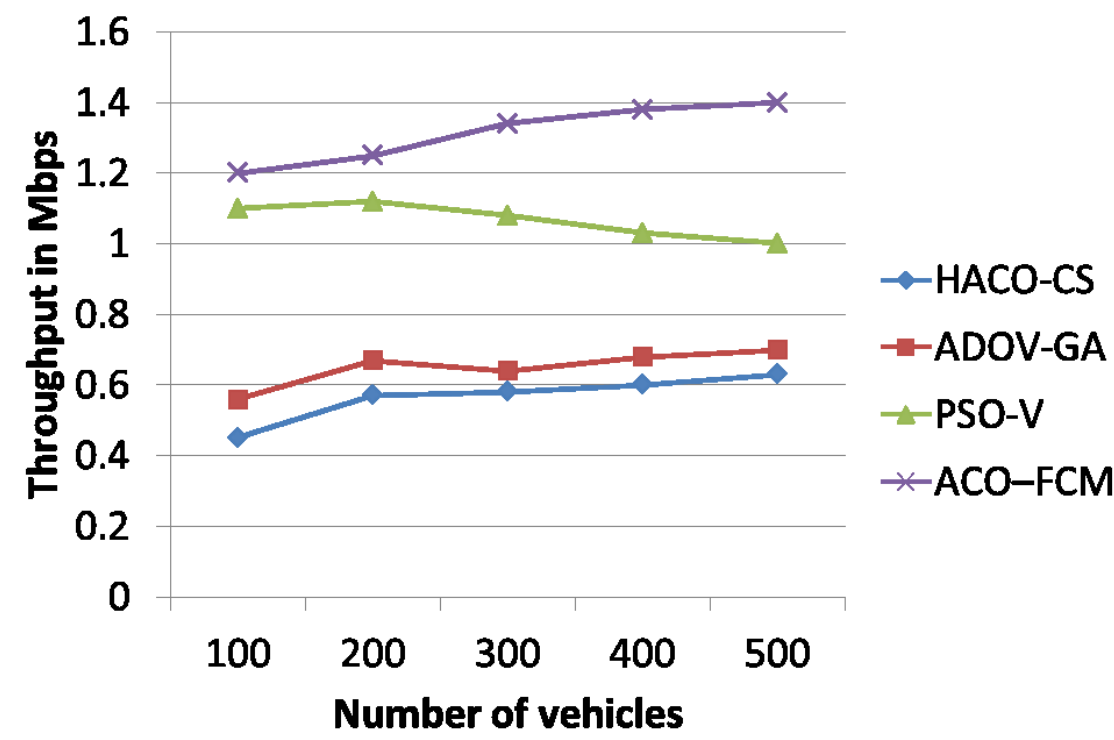

Fig.2.Throughput

The fig. 3 below shows the average time taken by the packets to travel through the network, the delay in the vehicular network includes the total delay that is comprised of the delay in the source to cluster-head transmission, initiation of route discovery, waiting for the path identification, and the conveyance of the packet. The results obtained and the comparison with the prevailing methods shows that the ACO-FCM ensures a considerable reduction in the delay than the HACO-CS, ADOV-GA and PSO-V.

ISSN: 2582-2640 (online) 
Journal of Soft Computing Paradigm (JSCP) (2019)

Vol.01/ No. 02

Pages: $91-102$

http://irojournals.com/jscp/

DOI: https://doi.org/10.36548/jscp.2019.2.004

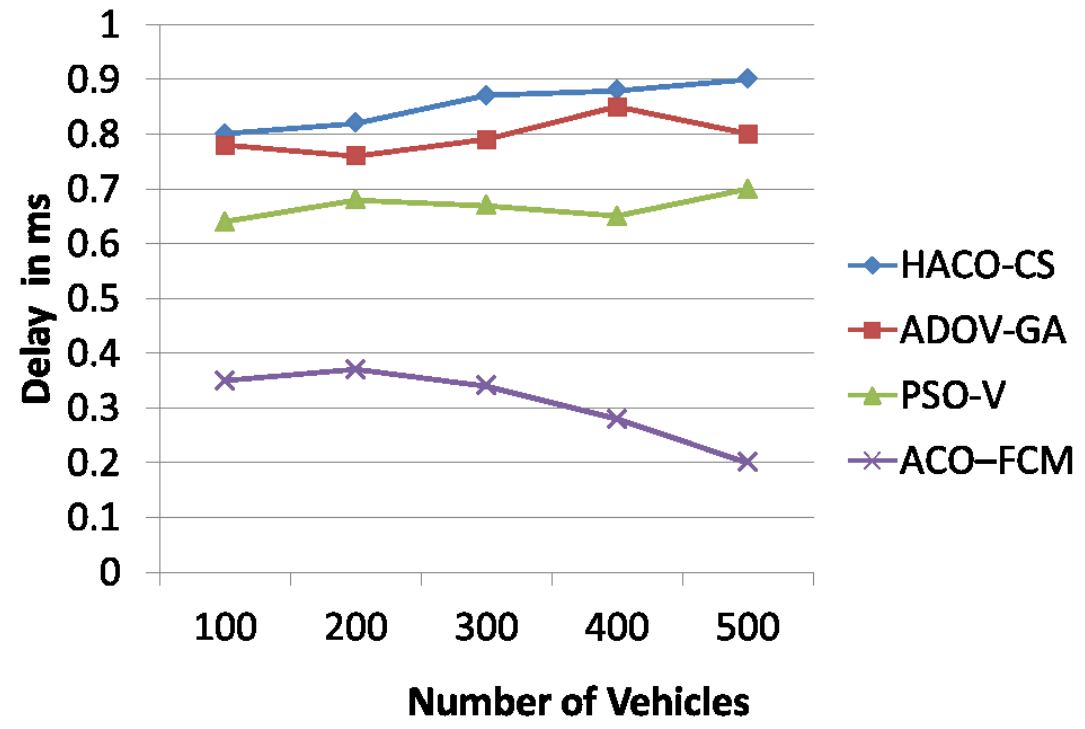

Fig.3 Delay

The Fig. 4 below shows the percentage of link life expectancy obtained for the proposed method along with the comparison with the HACO-CS, ADOV-GA and PSO-V.

ISSN: 2582-2640 (online)

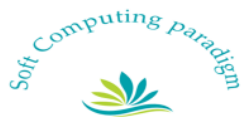


Journal of Soft Computing Paradigm (JSCP) (2019)

Vol.01/ No. 02

Pages: $91-102$

http://irojournals.com/jscp/

DOI: https://doi.org/10.36548/jscp.2019.2.004

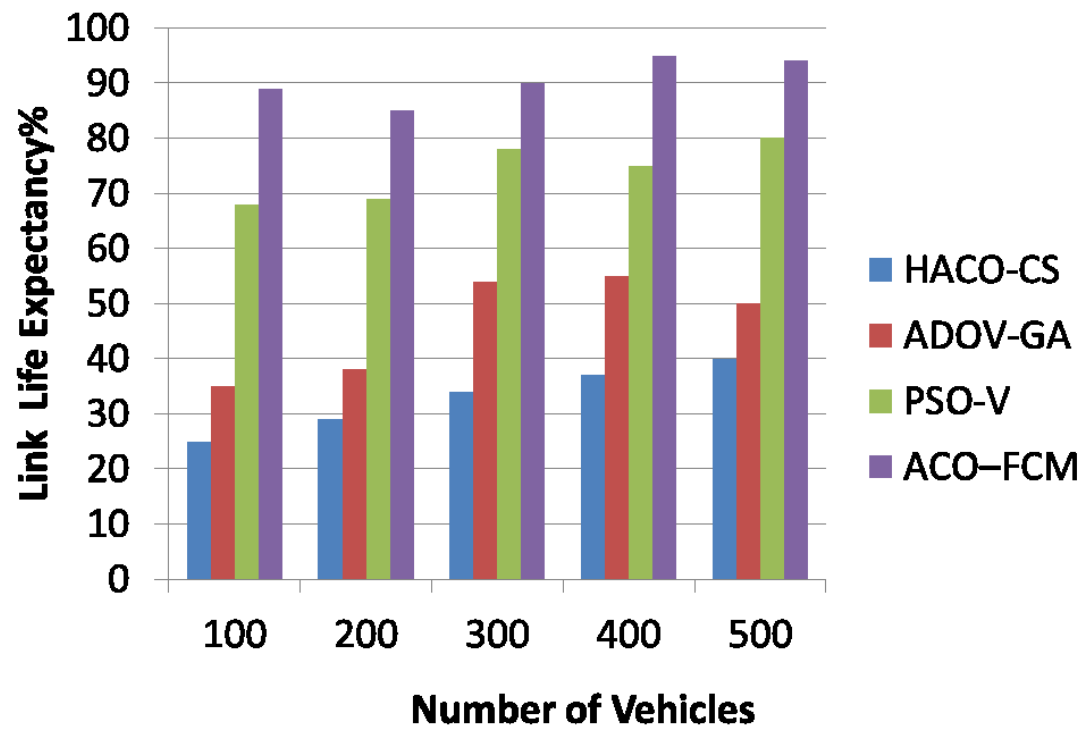

Fig.4. Link Life Expectancy

\section{CONCLUSION}

The Vehicular network is a wireless communication network that is framed specifically to improve the convenience as well as the safety in on road transportation. It disperses the messages regarding the traffic and the disaster on the road to the vehicles using the other vehicles or the roadside unit. To avoid the delay in the communication and improve the quality of service of the VANET, the paper has proposed the ACO incorporated with the Fuzzy C means clustering enables to identify an QOS optimized path for the conveying of the information by proper cluster framing, cluster head selection using the fuzzy $\mathrm{C}$ means and engaging the ACO in identifying the path with the enhanced QOS metrics. The evaluation of the proposed method using the network simulator-2 proves the improved QOS attained in the VANET by the proposed method on comparing with the prevailing methods. The future aim of the paper is to secure the information transfer in the vehicular networks by identify the a QOS enhanced path enriched with the trusted nodes.

ISSN: 2582-2640 (online)

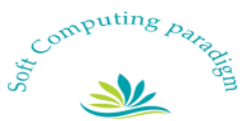


Journal of Soft Computing Paradigm (JSCP) (2019)

Vol.01/ No. 02

Pages: 91- 102

http://irojournals.com/jscp/

DOI: https://doi.org/10.36548/jscp.2019.2.004

\section{References}

[1] Zaghar, Dhafer R., and Thulfiqar S. Aldeen A. AL Wahab. "Simplified the QoS factor for the ad-hoc network using fuzzy technique." International Journal of Communications, Network and System Sciences 6, no. 09 (2013): 381.

[2] Toutouh, Jamal, and Enrique Alba. "Parallel swarm intelligence for VANETs optimization." In 2012 Seventh International Conference on P2P, Parallel, Grid, Cloud and Internet Computing, pp. 285-290. IEEE, 2012.

[3] Kaur, Shabad, and A. Virk. "AODV extension using genetic algorithm in VANET." International Journal of Science and Research 4, no. 7 (2015): 2420-2422.

[4] Sharawi, Marwa, Imane Aly Saroit, Hesham El-Mahdy, and Eid Emary. "Routing wireless sensor networks based on soft computing paradigms: survey." International Journal on Soft Computing, Artificial Intelligence and Applications (IJSCAI) 2, no. 4 (2013): 21-36.

[5] Jindal, Jaspal, and Vishal Gupta. "Fuzzy Improved Genetic Approach for Route Optimization in MANET." International Journal of Advanced Research in Computer Science and Software Engineering 3, no. 6 (2013).

[6] Giri, Parimal Kumar. "A Survey on Soft Computing Techniques for Multi-Constrained QoS Routing in MANET." International Journal of Computer and Information Technology (IJCIT), ISSN (2012): 22185224.

[7] Vijayakumar, V., P. Inbavalli, K. Suresh Joseph, J. Amudhavel, D. Rajaguru, S. Sampath Kumar, T. Vengattaraman, and K. Premkumar. "Research on QoS aware dynamic reconfiguration and performance measures in VANET." In 2015 Global Conference on Communication Technologies (GCCT), pp. 829-833. IEEE, 2015.

[8] Nancharaiah, B., and B. Chandra Mohan. "Hybrid optimization using ant colony optimization and cuckoo search in manet routing." In 2014 International Conference on Communication and Signal Processing, pp. 1729-1734. IEEE, 2014.

[9] Baskaran, Anush, Nikhil Chaudhari, Ronnie D. Caytiles, and N. C. S. Iyengar. "Fuzzy optimized and bee inspired routing protocol for improved qos in mobile ad hoc networks." International Journal of Control and Automation 9, no. 8 (2016): 391-402.

ISSN: 2582-2640 (online)

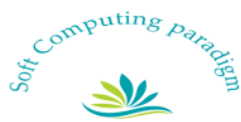


Journal of Soft Computing Paradigm (JSCP) (2019)

Vol.01/ No. 02

Pages: 91- 102

http://irojournals.com/jscp/

DOI: https://doi.org/10.36548/jscp.2019.2.004

[10] Benamar, Nabil, Kamal D. Singh, Maria Benamar, Driss El Ouadghiri, and Jean-Marie Bonnin. "Routing protocols in vehicular delay tolerant networks: A comprehensive survey." Computer Communications 48 (2014): 141-158.

[11] Kumar, Rakesh, and Mayank Dave. "A comparative study of Various Routing Protocols in VANET." arXiv preprint arXiv:1108.2094 (2011).

[12] Kaur, Kavneet, and Sandeep Kad. "A Study of Reliable Routing Protocols for Vehicular Ad hoc Networks." International Journal of Computer Applications 133, no. 3 (2016): 37-42.

[13] Bhalaji, N. "PERFORMANCE EVALUATION OF FLYING WIRELESS NETWORK WITH VANET ROUTING PROTOCOL." Journal of ISMAC 1, no. 01 (2019): 56-71.

[14] Raj, Jennifer S., S. Smys, and Abul Bashar. "ENERGY EFFICIENT MULTI-TIER SUSTAINABLE SECURE ROUTING PROTOCOL FOR MOBILE WIRELESS SENSOR NETWORKS."

[15] Hoang, Duc Chinh, Rasjesh Kumar, and Sanjib Kumar Panda. "Realisation of a cluster-based protocol using fuzzy C-means algorithm for wireless sensor networks." IET Wireless Sensor Systems 3, no. 3 (2013): 163-171.

ISSN: 2582-2640 (online)

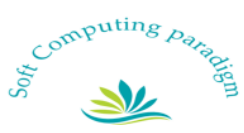

